\title{
Formalin needle disinfection reduces sepsis risk of prostate biopsy
}

New data suggest that disinfecting the biopsy needle with formalin after each prostate biopsy core is taken reduces the risk of urinary infection and sepsis, with minimal potential harm to the patient.

Issa et al. examined 1,642 consecutive patients with prebiopsy negative urine culture who underwent prostate biopsies from 2008 to 2012. Formalin (10\%) was used to disinfect the needle after each biopsy core and all patients were monitored for postbiopsy infection. The overall rate of urinary infection and sepsis was just $0.3 \%$, compared with $0.8 \%$ in a prior series of 990 procedures.

Ex vivo studies confirmed the efficacy of formalin against fluoroquinolone-resistant E. coli and showed that total formalin volume exposure after 12 biopsy cores was $0.039 \mathrm{ml}$ ( $3.9 \mathrm{mg}$ formaldehyde), which is a fraction of the daily safe limit set by the Environmental Protection Agency (EPA). "Putting it into perspective, the EPA considers it safe to be exposed to 1,000 times that amount of formalin every year," says Muta Issa, who led the study.
At present, increasingly powerful prophylactic antibiotics are given to reduce biopsy risk, but this is a shortterm reactive solution. In addition to the associated cost, inconvenience, and adverse effects, the use of increasingly powerful antibiotics is likely to promote the development of resistant and lethal bacteria. "If continued, it only a matter of time before we get a strain of bacteria that is highly resistant to all antibiotics," continues Issa.

As well as being safe, the formalin needle disinfection technique is simple, effective, and cost-neutral. However, the authors are keen to emphasize that formalin is just one of many interventions that should be used to lower infection risk. They also recommend that patients receive a bisacodyl enema and fluoroquinolone antibiotics, and that clinicians use a single-use bacteriostatic lubricant, a no-touch technique, and follow strict protocols that have been approved by infection disease experts for cleaning and sterilizing instruments.

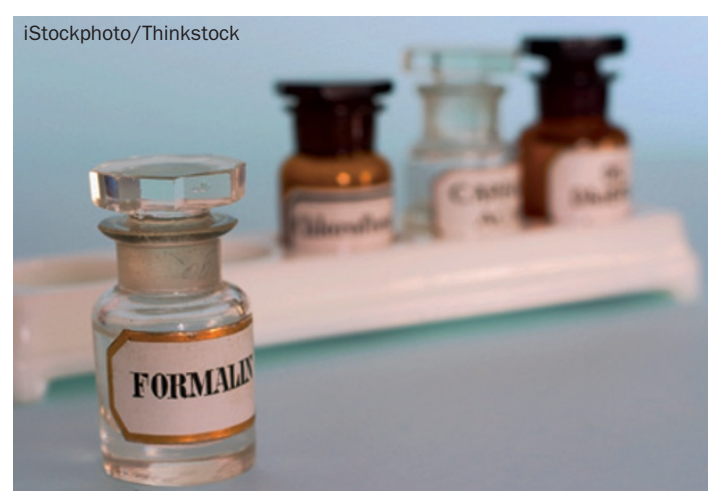

“Techniques such as disinfecting the biopsy needle between biopsy core acquisitions seem feasible but need to be reproduced in contemporary randomized trials," warns Nathan Lawrentschuk, a leading expert in the field.

Melanie Clyne

Original article Issa, M. M. et al. Formalin disinfection of biopsy needle minimizes the risk of sepsis following prostate biopsy. J. Urol. doi:10.1016/j.juro.2013.04.134 